\title{
La Lusace, pays d'étangs : usages traditionnels de l'eau et nouvelles pratiques en territoires sorabes (Länder de Brandebourg et Saxe, Allemagne).
}

The Lusatia, region of ponds : traditionnal uses and of water and new practices in sorbian territories (Länder of Brandenburg and Saxony, Germany)

Die Lausitz, ein Teichland : traditionnelle Verwendungen des Wassers und neue Praktiken in sorbischen Gebieten (Länder von Brandenburg und Sachsen)

\section{Anatole Danto}

\section{OpenEdition}

\section{Journals}

Electronic version

URL: http://journals.openedition.org/rge/5739

ISSN: $2108-6478$

Publisher

Association des géographes de l'Est

Printed version

Date of publication: 22 April 2016

ISSN: 0035-3213

Electronic reference

Anatole Danto, «La Lusace, pays d'étangs : usages traditionnels de l'eau et nouvelles pratiques en territoires sorabes (Länder de Brandebourg et Saxe, Allemagne). », Revue Géographique de l'Est [Online], vol.56 / n¹-2 | 2016, Online since 29 June 2016, connection on 08 September 2020. URL : http:// journals.openedition.org/rge/5739

This text was automatically generated on 8 September 2020

Tous droits réservés 


\section{La Lusace, pays d'étangs : usages traditionnels de l'eau et nouvelles pratiques en territoires sorabes (Länder de Brandebourg et Saxe, Allemagne).}

The Lusatia, region of ponds : traditionnal uses and of water and new practices in sorbian territories (Länder of Brandenburg and Saxony, Germany)

Die Lausitz, ein Teichland : traditionnelle Verwendungen des Wassers und neue Praktiken in sorbischen Gebieten (Länder von Brandenburg und Sachsen)

\section{Anatole Danto}

1 Le sud du land du Brandebourg (Brandenburg en allemand, Braniborska en sorabe) et l'est $\mathrm{du}$ land de Saxe (Sachsen, Sakska) forment la région allemande de Lusace (Cf. carte 1). Situé aux confins nord-est du pays, aux frontières de la Pologne et de la République Tchèque, cet espace, autrefois intégré à l'Allemagne de l'Est, est peuplé depuis de nombreux siècles par une minorité slave: les Sorabes. La Lusace est un territoire globalement peu accidenté, et est drainé par un dense réseau hydrographique. Les populations établies ont depuis longtemps tiré profit de ce réseau, en créant par exemple des étangs, notamment utilisés pour la pisciculture. Connue pour sa production de cornichons à l'époque de la $\operatorname{RDA}^{1}$ (Spreewaldgurken, indication géographique protégée depuis 1999), la région l'est également pour ses lieux de pêche, ses poissons d'eau douce, ou son patrimoine lié aux usages vivriers de l'eau par les Saxons et les Sorabes. Ce dernier est d'ailleurs mis à contribution pour promouvoir cet espace auprès des touristes allemands, voire étrangers. Les objectifs de l'article sont d'exposer quels sont les différents usages traditionnels de l'eau en Lusace, mais aussi de montrer quelles fonctionnalités nouvelles émergent dans cette région d'étangs. 


\section{I - Présentation géo-historique de la Lusace}

2 La Lusace, vaste de près de 5000 kilomètres carrés, borde les frontières germanopolonaise et germano-tchèque. Elle est peuplée depuis 1400 ans par une minorité ethnique, les Sorabes, peuple d'origine slave installé sur ce territoire en cohabitation avec un peuple germanique, les Saxons. L'influence des Sorabes s'étendait auparavant bien au-delà des frontières de l'actuelle Lusace, comme le montre l'origine du nom Leipzig ("lieu près des tilleuls").

\section{A - Historique du peuplement et aspects minoritaires}

3 Le substantif «Sorabes" (Sorben en allemand) est employé pour désigner les descendants de peuplades slaves, établies dans l'est de l'actuelle Allemagne aux environs du VIème siècle. D'autres termes désignent également ces populations, tels que "Serbes de Lusace», ou "Wendes » (terme plus large faisant référence aux tribus slaves installées entre l'Elbe et la Vistule). Les Sorabes, peuple frontalier, auraient colonisé la Lusace "par dépit", repoussés dans la zone-refuge des marais lusaciens (Koter, 1995).

4 La Lusace est scindée en deux régions principales d'un point de vue ethnique : la BasseLusace et la Haute-Lusace. La première, située au nord de la Lusace, est peuplée par des Sorabes protestants, et est plus accidentée, tandis que la seconde, au sud, l'est par des Sorabes catholiques. Cette dichotomie, qui constitue pour nombre d'auteurs un dualisme historique (Yèche, 2014), est d'ailleurs sources de nombreuses différences ethnologiques et sociologiques. On estime à environ 40000 le nombre de locuteurs Sorabes encore présents en Lusace, avec toutefois une différence entre la Haute-Lusace, où est parlé le haut-sorabe (environ 30 000), et la Basse-Lusace, où l'on s'exprime en bas-sorabe (près de 10 000). Ces deux langues Sorabes, proches, connaissent aussi des différences dialectales internes, notamment en haut-sorabe, ainsi qu'un dialecte dit de transition, entre la Haute et la Basse-Lusace (Sanguin, 1996). Elles sont protégées par l'État allemand et les Länder où elles sont parlées, notamment au travers de la signature de la Charte européenne des langues régionales ou minoritaires, au même titre que les langues des deux autres minorités allemandes reconnues comme telle : les Frisons et les Danois du Schleswig (Conseil de l'Europe, 2004). Il existe plusieurs écoles bilingues, et le sorabe est également enseigné jusqu'à l'université, avec un département de sorabistique à l'université de Leipzig². Des lois et arrêtés régionaux pris par la Saxe ou le Brandebourg viennent aussi renforcer la protection réglementaire de la culture sorabe. Signalons également que pendant la RDA, la grande majorité des traditions culturelles sorabes n'ont pas été contraintes par l'État, qui a tout-de-même exercé un important contrôle sur la minorité (Kudela, 1985).

\section{B - Géographie et hydrographie régionales}

5 La Lusace est appelée Lausitz en allemand, Łužica en haut-sorabe, Łužyca en bas-sorabe. Etymologiquement, son nom signifie en sorabe "lieu riche en eau" (Kowar, 1994). Elle est scindée en plusieurs ensembles géographiques distincts. La Haute-Lusace, située au sud de la région, près de la frontière tchèque, est très vallonnée à son extrême sud, aux alentours des Monts de Zittau (début de la chaîne des Sudètes), des Monts de Lusace et 
des Monts de la Jizera, avec des hauteurs conséquentes (700 mètres), et la naissance de plusieurs cours d'eau d'importance, comme la Neisse sur le territoire tchèque, ou la Spree. L'ensemble a subi une forte déforestation et est aujourd'hui cultivé.

Plus au nord, au-delà de la ville-centre de Bautzen (Budyšin, 40000 habitants environ), capitale de la Haute-Lusace, se trouve la région des landes et étangs de Haute-Lusace (Oberlausitzer Heide- und Teichlandschaft), bien moins cultivée, plus boisée, jalonnée d'étangs et de cours d'eau, et classée comme réserve de biosphère de l'UNESCO ${ }^{3}$. Cette région est surnommée localement "le pays aux mille étangs", comme beaucoup d'autres régions européennes et notamment françaises (Saône, Brenne, etc). Certains de ces étangs ont été créés au cours du Moyen-Âge, d'autres plus tardivement (XVI ${ }^{\text {ème }}$ siècle, années 1950), et ont souvent été remaniés.

$7 \quad$ La frontière entre Haute et Basse-Lusace se situe aux environs de Weißwasser (Béła Woda, 20000 habitants), dans le Seenland (pays des lacs). Le paysage est alors marqué par les importantes mines d'extraction de lignite ou d'alun. Plusieurs d'entre elles, désaffectées, on été mises en eau depuis les années 1950, et un nouveau plan d'inondation est prévu jusqu'en 2018, devant toucher près d'une vingtaine de sites. En parallèle, la production électrique à partir de charbon redevient compétitive en Allemagne. Le Land de Saxe a ainsi autorisé l'extension ou la réouverture de plusieurs mines de lignite pour accroître les capacités de production, déplaçant au passage des villages entiers peuplés de Sorabes et faisant craindre des menaces sur leur patrimoine (Daiber, 2014).

8 Enfin, la Basse-Lusace, vaste espace dont la ville-centre est Cottbus (Chóśebuz, 102000 habitants), est une région plane s'étendant autour du lit de la Spree, appelée Spreewald (Błota), correspondant à une immense plaine d'inondations naturelle, inondations qui peuvent être de forte intensité. La rivière, avant de rejoindre la Havel (le débit moyen de la Spree y est alors d'environ $36 \mathrm{~m}^{3} . \mathrm{s}^{-1}$, mais peut monter au-delà de 100 lors des crues importantes), serpente ici au milieu des forêts, et comporte, dans son lit majeur, de nombreux bras annexes (plusieurs centaines). L'hydronyme Spree est d'origine slave, signifiant "la rivière sorabe", preuve de son importance pour la région. La BasseLusace historique s'étend à l'est jusqu'à l'Oder, au nord jusqu'à une ligne reliant le sud de Frankfurt-an-der-Oder et la ville de Baruth, à une quarantaine de kilomètres au sud de la capitale allemande, et enfin à l'ouest jusqu'à la rivière Elster, affluent de l'Elbe. 
Carte 1 : Carte de localisation : géographie de la Lusace, sources : Geofabrik $\mathrm{GmbH}$, Agence européenne de l'environnement, Union Européenne.

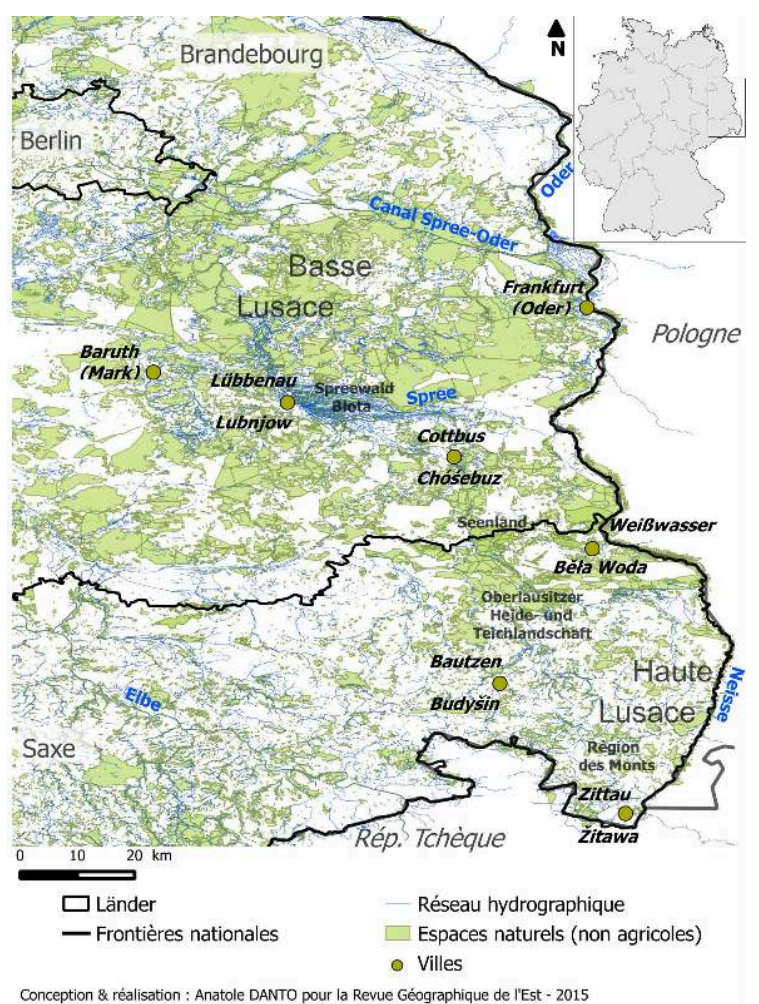

9 La Lusace est donc une région essentiellement rurale, même s'il existe de très anciennes villes faisant office de lieux centraux pour le territoire. Le paysage est essentiellement agro-forestier, mais est aussi organisé autour du dense réseau hydrographique, des étangs et canaux, et des mines. La ligne de partage des eaux entre le bassin versant de l'Oder (Baltique) et celui de l'Elbe (Mer du Nord) traverse également la Lusace.

\section{II - Les usages traditionnels de l'eau en territoire sorabe}

10 Cette hydrographie et le peuplement ancien en présence sur le territoire lusacien ont bien évidemment conduit les Sorabes à utiliser l'eau depuis toujours. De nombreux usages traditionnels sont ainsi encore visibles aujourd'hui, ou ont laissé des traces, notamment dans l'onomastique locale et plus particulièrement l'hydronymie et la toponymie (Duma \& Rzetelska-Feleszko, 1974).

\section{A - Les traditions liées à l'eau : fêtes, coutumes et symbolique}

11 L'année sorabe est jalonnée d'événements en lien avec des coutumes traditionnelles souvent proches de la nature. Parmi celles-ci, plusieurs sont liées à l'eau et aux milieux aquatiques. Au mois de février a lieu le carnaval sorabe et son mardi gras (Zapust), qui dure une semaine. Pendant cette période se déroule le cortège (Camprowanje), inspiré de rites païens, qui a pour but de chasser les mauvais esprits et de protéger la communauté. Des baguettes d'osier, arbuste ligneux emblématique des zones humides, 
sont alors utilisées en grande quantité pour effrayer les démons, et appelées "baguettes de vie" (Scholze, 2011). Dans le même temps, le retour de la cigogne dans les marais symbolise aussi l'arrivée du printemps. La symbolique de l'imposant oiseau était souvent utilisée pour les déguisements traditionnels lors du carnaval.

12 Aux alentours de Pâques, un autre oiseau des régions palustres a une place dans les traditions locales : l'oie, qui était souvent élevée auparavant. Les plumes les plus fines de l'anatidé sont taillées minutieusement pour être utilisées pour peindre les œufs de Pâques et appliquer de la cire dessus pour créer des motifs. Les œufs ainsi décorés serviront à embellir les villages à l'approche de la fête pascale ou seront offerts à des proches. Toujours en lien avec cette fête, l'eau de Pâques était traditionnellement recherchée par les Sorabes, qui se lavaient avec et arrosaient leur bétail (Tschernokoshewa, 2011). Dans la nuit du samedi au dimanche de Pâques, les jeunes filles allaient chercher l'eau dans les rivières où elle s'écoule de l'est vers l'ouest (comme le soleil), reconnue pour ses vertus curatives (Jatšowna woda). Si les jeunes femmes parlaient en ramenant l'eau de la rivière, celle-ci perdait ses vertus, et devenait alors de "l'eau bavarde" (Kuringowa, 2005). Certaines plantes aquatiques ont aussi une place particulière dans l'imaginaire collectif de Lusace, comme le nénuphar, qui symbolise l'eau comme source de vie. Il est d'ailleurs utilisé pour fabriquer la couronne de celui qui jouera le rôle de Jean lors des chevauchées de la Saint-Jean (Jańske rejtowanje).

13 Cependant, certaines traditions passées ou actuelles de Lusace n'ont pas que des origines chrétiennes (catholiques ou protestantes), mais aussi païennes, et notamment saxonnes. Dans les anciennes religions saxonnes, polythéistes, il existait en effet des divinités liées à l'eau ou à la pêche, célébrées il ya longtemps en Lusace, comme le dieu Krodo, symbolisé par un vieillard, debout sur un poisson (une perche). D'autres esprits animaient aussi l'imaginaire commun, à l'image de Nykus, l'homme de l'eau (Wassermann), qui habite les fonds de l'eau (Neumann, 2009).

14 L'eau revêtait, et revêt encore une place importante dans la culture sorabe, notamment à travers l'environnement aquatique présent en Lusace (faune, flore, ...). Mais l'eau connaît aussi des usages quotidiens matériels dans la région.

\section{B - Usages énergétiques et de transport.}

15 En Lusace, la topographie et l'hydrologie ont très tôt permis l'apparition de moulins utilisant l'énergie hydraulique, avec créations de biefs et de retenues artificielles (étangs), ou de canaux de détournement de l'eau. De nombreux moulins jalonnent ainsi le réseau de petits cours d'eau, plusieurs d'entre eux étant encore en activité, et font l'objet de promotion touristique et mise en valeur patrimoniale. Certains datent $\mathrm{du}$ XIIIème siècle, et servaient au départ à la meunerie des villages de Lusace (production de farine). L'agglomération de Cottbus par exemple, compte à elle seule une dizaine de moulins, dont plusieurs fonctionnent encore (mais parfois à l'énergie électrique). Dans toute la Lusace, une quarantaine de moulins est encore inventoriée par l'association allemande de préservation des moulins (Deutsche Gesellschaft für Mühlenkunde und Mühlenerhaltung ${ }^{4}$ ), avec une prédominance de moulins à eau en aval de Cottbus, soit en Basse-Lusace.

16 L'eau a aussi permis, comme dans de nombreuses régions d'Europe, une protoindustrialisation du territoire avant la révolution industrielle. En plus des moulins, 
l'eau a été utilisée pour fournir de l'énergie à une importante industrie locale, notamment des filatures. Aujourd'hui encore, l'eau fournit de l'énergie à des entreprises lusaciennes : papeteries, usines métallurgiques, mais surtout industrie chimique. En matière de transport, l'eau a également été un vecteur important du développement de ce secteur dans la région, en permettant d'accroître les échanges. Le transport de marchandises par des moyens nautiques de petite taille et sur de courtes distances a été important sur les cours d'eau locaux. De nombreuses écluses, modestes en taille, ponctuent ainsi le réseau. Au nord de la Lusace, la Spree rejoint le canal SpreeOder, qui est inscrit comme voie navigable nationale, et gérée par le WSA de Berlin ${ }^{5}$. Enfin, localement, les canaux, appelées Fließe, sont utilisés pour des besoins précis, comme la distribution du courrier par la poste dans la ville de Lübbenau par exemple ${ }^{6}$.

\section{C - Pêche et aquaculture : une exploitation intensive des ressources piscicoles}

17 Toutefois, l'usage de l'eau le plus important et le plus visible aujourd'hui en Lusace est l'aquaculture, et plus particulièrement la pisciculture, issue de pratiques traditionnelles remontant à l'époque médiévale (Hartstock, 2000). La région est en effet l'une des premières régions d'Allemagne dans le domaine de la production piscicole, au coude à coude avec les lacs du Mecklembourg (Land du Mecklenburg-Vorpommern). De très nombreux étangs ont ainsi été creusés et mis en eau pour accueillir de la pisciculture. Les poissons tiennent d'ailleurs une place importante dans la culture sorabe, et certains ichtyonymes allemands sont issus de noms Sorabes, comme la loche, Schlammpeitzger, du sorabe piskor (Österreichische Fischereigesellschaft, 2006).

18 Trois grandes périodes de création d'étangs se sont succédées en Lusace ( $C f$. carte 2). La première mention de l'existence d'étangs en Lusace date de 1248, et provient de l'acte de fondation du cloître de Sankt Marienstern/Marijina Hwězda, dans la région de Bautzen (Füllner, 2000). Sous l'impulsion de familles nobiliaires ou de congrégations religieuses, des étangs sont aménagés dans les fonds de vallée de Haute-Lusace, créant de véritable lignes d'étang qui suivent la topographie locale des talwegs lusaciens. A proximité de ces étangs, et à la même période, des moulins à eau commencent à faire leur apparition, le long de Graben (fossés). Moulins et étangs, qui appartiennent au clergé ou à la noblesse sont ainsi édifiés pour permettre une production primaire locale, et ainsi récupérer des taxes lors de leur utilisation par les villageois. Les religieux surtout permettent des progrès techniques et technologiques en matière d'aquaculture, et ce comme dans le reste de l'Europe.

19 La seconde période de création d'étangs s'étend de la révolution industrielle aux années 1970. Elle est essentiellement le fait d'une volonté économique de la part tout d'abord d'entrepreneurs (qui y voyaient aussi un site de loisir pour la pêche), puis de l'administration socialiste est-allemande, qui encourageait fortement leur création. Les lieux choisis sont soit isolés (des prairies inondables par exemple), soit regroupés à proximité des marais de la Spree. La Basse-Lusace est alors plus concernée. La taille des étangs augmente progressivement au temps de la RDA, pour atteindre parfois plusieurs hectares.

20 La troisième, et pour l'instant dernière phase est marquée par la création de vastes plans d'eau dans d'anciennes mines de lignite, en voie d'ennoiement depuis l'arrêt de l'exploitation. Cette phase a débuté dans les années 1970 et s'est accélérée après la 
Réunification allemande et la fermeture de la plupart des exploitations de la région. Ces plans d'eau sont de très grande taille (parfois plus de 1000 hectares), et leur aménagement est plus long (dépollution, creusement des berges dans la roche, ...). Ils sont essentiellement concentrés là où se trouvaient, et se trouvent encore les gisements de lignite, et sont donc organisés géographiquement le long des gisements dont l'exploitation est terminée, notamment à l'est et au sud de Cottbus. A terme, cette région doit devenir le plus vaste espace de lacs artificiels d'Europe.

Carte 2 : Carte de la typologie des étangs de Lusace, sources : Geofabrik $\mathrm{GmbH}$, Agence européenne de l'environnement, Union Européenne, WSA, UNESCO, données personnelles.

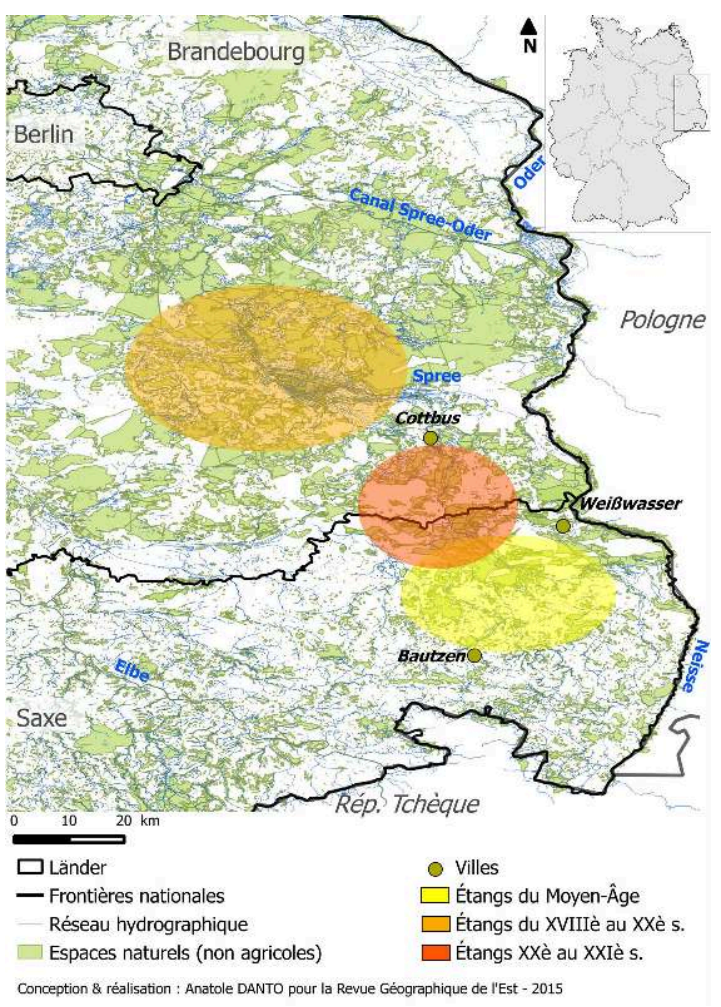

Tableau 1 : Typologie diachronique des étangs de Lusace.

\begin{tabular}{|c|c|c|c|c|c|c|}
\hline Date de création & Taille & Localisation & Environnement & Usages originels & Créateur & Usages contemporains \\
\hline Moyen-âge & $\begin{array}{l}\text { Petite } \\
\text { (< à } 1 \text { ha) }\end{array}$ & Haute-Lusace & \begin{tabular}{|l|}
$\begin{array}{l}\text { Fonds de vallée, } \\
\text { petits cours } \\
\text { d'eau }\end{array}$ \\
\end{tabular} & \begin{tabular}{|l|} 
- Petite pisciculture \\
-Energie hydraulique
\end{tabular} & $\begin{array}{l}\text { - Nobles } \\
\text { - Clergé }\end{array}$ & $\begin{array}{l}\text { - Réserves naturelles } \\
\text {-Abandon }\end{array}$ \\
\hline XVIII ${ }^{\text {ime }}$ s. - 1970 & $\begin{array}{l}\text { Moyenne } \\
\text { (1 à } 100 \text { ha) }\end{array}$ & Basse-Lusace & 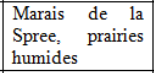 & $\begin{array}{l}\text {-Pêche de loisir } \\
\text { - Pisciculture }\end{array}$ & \begin{tabular}{|l|} 
- Bourgeois \\
-État est-allemand
\end{tabular} & - Pisciculture légère \\
\hline $1970-2020$ & $\begin{array}{l}\text { Grande } \\
(\ngtr \mathrm{a} 100 \mathrm{ha})\end{array}$ & Haute-Lusace & $\begin{array}{l}\text { Anciennes } \\
\text { mines de lignite }\end{array}$ & \begin{tabular}{|l|} 
- Pisciculture \\
- Loisirs nautiques et \\
balnáaires \\
- Espaces naturels \\
\end{tabular} & $\begin{array}{l}\text { - Entreprises } \\
\text { privées } \\
\text {-Länder }\end{array}$ & $\begin{array}{l}\text {-Pisciculture d'importance } \\
\text { - Loisirs } \\
\text { - Réserves naturelles }\end{array}$ \\
\hline
\end{tabular}

21 La principale espèce élevée dans les étangs lusaciens est la carpe. Pendant la RDA, son élevage a été intensifié, avec un accroissement important des capacités de production, atteignant ainsi près de 7000 tonnes par an en Saxe en 1989. Depuis cette date, la production oscille entre 1700 et 2800 tonnes suivant les années (Füllner, Bild \& Matko, 2013), l'Allemagne restant le septième producteur européen de carpes. A la chute de la RDA, les coopératives de pêcheurs et les fermes aquacoles ont été décollectivisées, et se sont transformées juridiquement pour entrer dans l'économie de marché. Nombre d'entre elles ont alors fait face à d'importantes difficultés financières, corrélées à une chute de la commande publique, mais aussi à la fin de la planification économique 
(objectifs de production à atteindre). D'autres poissons sont aussi élevés dans la région, comme la truite (300 à 400 tonnes par an), ou des productions de poissons exogènes, plus anecdotiques, comme le tilapia, même si ces dernières sont en forte augmentation (passage de 38 tonnes en 2004 à 419 tonnes en 2013) (Füllner, Bild \& Matko, op. cit.).

22 La pêche, quant à elle, est pratiquée à la fois par des pêcheurs amateurs et des pêcheurs professionnels. Les premiers pêchent essentiellement à la ligne, tandis que les seconds pratiquent la pêche aux engins et aux filets, avec surtout la pose de nasses ou de filets tramail à plusieurs nappes. Les principales espèces ainsi capturées sont la tanche, la carpe, le brochet, le sandre, la truite et la lotte. Les ressources en poissons sauvages sont, d'après certains pêcheurs, en diminution, faute à la pollution avérée des eaux par l'agriculture et l'industrie lourde, et à la discontinuité écologique des cours d'eau, qui affectent notamment les stocks de lottes (Brotfisch) ou d'anguilles (Aal). Les captures totales des pêcheurs sont ainsi passées de 220 tonnes en 1995 à 160 tonnes en 2010 (Füllner, Bild \& Matko, op. cit.). La rupture à la continuité écologique est petit à petit réduite par l'intervention des pouvoirs publics, qui cherchent à se conformer à la réglementation communautaire (Directive Cadre sur l'Eau notamment). Pour pêcher en Lusace, il faut être titulaire de droits de pêche, et adhérer à une association, à l'image de la société wende/sorabe des pêcheurs de la forêt de la Spree de Burg et de ses environs (Gemeinschaft wendisch/sorbischer Spreewaldfischer Burg und Umgebung) ${ }^{7}$, qui regroupe 160 pêcheurs, répartis en 17 comités.

23 L'aquaculture et la pêche tiennent donc une place importante dans l'économie locale de la Lusace. La pêche est plutôt pratiquée en Basse-Lusace, dans les marais de la Spreewald, tandis que l'aquaculture est plus importante en Haute-Lusace. Les poissons sont pêchés et élevés dans cette région depuis plusieurs siècles, avec une intensification récente de la production en étangs (une cinquantaine d'années), critiquée à la chute de la RDA pour son impact environnemental, moins aujourd'hui. Les Länder de Saxe et du Brandebourg sont parmi les premiers d'Allemagne en terme de consommation de poissons $(5.7 \mathrm{~kg} / \mathrm{an} / \mathrm{hab})$, juste après trois Länder littoraux.

\section{III - L'émergence de nouveaux usages}

24 La période de la RDA a eu d'importantes conséquences sur la région. En matière d'aquaculture, on l'a vu, le régime socialiste a engagé un vaste plan d'augmentation spatiale et quantitative de la production piscicole. Mais en Lusace, le plus marquant a été l'exploitation de lignite à grande échelle, véritable source énergétique pour toute l'Allemagne de l'est, avec l'ouverture d'immenses mines, pourvoyeuses d'emplois (de nombreux Allemands germaniques, et non sorabes, furent installés en Lusace lors de cette période, participant indirectement à une politique d'assimilation de la minorité, cf. Marti, 2005). L'hydrologie de la région a été bouleversée par cette industrie minière, avec notamment une pollution accrue des cours d'eau, mais aussi de vastes travaux de rectification de cours d'eau, de mise en réseau d'étangs, de création de retenues, de détournements de lits, etc.. Lors de la réunification, la Lusace entre, avec le reste de la RDA, dans l'économie de marché, et entame alors une profonde mutation économique, qui se traduit par un marasme au début des années 1990, la suppression de nombreux emplois dans les mines, et l'écroulement de la production aquacole. Au début des années 2000, en parallèle de ces usages traditionnels et anciens de l'eau, d'autres 
formes d'usages commencent à émerger en Lusace, comme les usages patrimoniaux, touristiques et environnementaux.

\section{A - Une patrimonialisation accrue des usages de l'eau source d'apports touristiques}

En effet, la région de Lusace s'est engagée depuis quelques années dans le développement touristique du territoire, profitant notamment de la manne financière des exploitations minières. Les mines de lignite emploient en effet de nombreux travailleurs, utilisent des services secondaires et tertiaires importants (transport par exemple), et ont une politique de verdissement de leur image, qui passe notamment par le financement d'actions de "développement durable" localement (notamment environnementales mais aussi sociales). La Lusace tire également profit des possibilités offertes par les programmes européens : FEDER, LEADER, LIFE, etc (Entwicklungsprogramm für den ländlichen Raum in Freistaat Sachsen 2007-2013, 2011).

Les usages de l'eau, essentiellement les transports par voie d'eau, les fêtes traditionnelles et la pêche, sont mis en avant par les collectivités locales. Emergent ainsi des lieux ou des événements dédiés à montrer au public la richesse du patrimoine lié à l'eau en Lusace. Le réseau hydrographique est utilisé aujourd'hui pour des tours en bateau touristique, à l'heure, à la journée, voire plus, au travers des canaux de la Spree notamment, et avec une jonction vers le canal Spree-Oder, qui remonte jusqu'à Berlin. Les coutumes traditionnelles comme les fêtes et les croyances sont aussi remises au goût du jour à travers toute la Lusace, à l'image des anciens dieux saxons, dont des sculptures les représentant servent aujourd'hui d'attractions à l'Erlebnispark Teichland deNeuendorf (Brandebourg), ou encore aux célébrations pascales en zones urbaines comme en zones rurales.

27 Mais c'est surtout sur les poissons que mise la région aujourd'hui, en visant un public averti, intéressé par la quantité et la qualité des milieux aquatiques lusaciens. Ainsi, les organisations touristiques locales (offices, associations, ...) ont créé "les semaines lusaciennes du poisson", qui ont lieu chaque année depuis 14 ans, et qui regroupent une quarantaine de partenaires. 
Image 1 : affiche de l'édition 2014 des semaines lusaciennes du poisson (Lausitzer Fischwochen), source : www.lausitzer-fischwochen.de

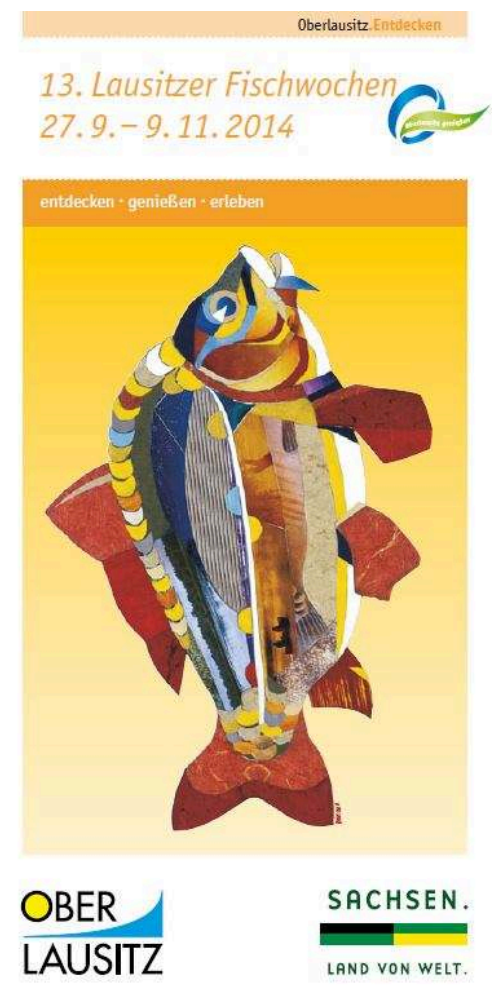

On peut alors pratiquer différentes techniques de pêche, visiter des fermes aquacoles, ou déguster des produits régionaux à travers toute la Lusace. Chaque édition s'étend sur un mois et demi, de la fin septembre à la mi-novembre, et draine de nombreux amateurs. Des formules clefs en main sont aussi proposées sur plusieurs jours lors de cet événement, pour faire découvrir les milieux aquatiques régionaux aux touristes (comprenant l'hébergement, la restauration, et des visites locales), tout comme des "tours touristiques et gastronomiques du pays" (Landtouristisch-kulinarische Touren).

Enfin, la patrimonialisation des usages de l'eau en pays sorabe passe aussi par les musées (et notamment les Heimatstube - musée local - et Freilichtmuseum - écomusée, dont certains sont spécialisés sur la pêche, comme le Fischereimuseum de Peitz ${ }^{8}$ ), ou encore par les maisons de la nature (maison de site, de parc, de réserves de biosphère, etc), avec découverte de l'environnement local.

La Lusace est donc lancée dans une démarche de mise en avant du patrimoine rural de sa région, dans le but d'attirer de nouveaux visiteurs. Toutefois, plusieurs critiquent pourraient être formulées, notamment sur l'origine de cette patrimonialisation, parfois n'émanant pas des Sorabes mais s'intéressant à leur territoire et à leur culture : certains profitent des retombées économiques du tourisme en patrimonialisant certaines activités traditionnelles ou certaines fêtes et coutumes, pas forcément vues comme un patrimoine par les sorabes (la notion même de patrimoine reste une vaste question, surtout lorsque l'on s'intéresse au patrimoine culturel immatériel) (UNESCO, 2011). L'augmentation du nombre de touristes, vue comme une aubaine économique pour le territoire n'est donc pas sans poser différentes questions dans cet espace rural. 


\section{B - Labellisation des productions et préservation de l'environnement}

Toutefois, en parallèle de cette démarche, le territoire lusacien cherche aussi à tirer partie de son patrimoine naturel, avec là encore, la mise en place de différentes actions.

Les pêcheurs professionnels ou amateurs, aidés en cela par les administrations locales, comme le Landesamt für Umwelt, Landwirtschaft und Geologie en Saxe, se sont lancés dans des programmes de ré-empoissonnement, avec marquage et suivi d'espèces, mais aussi de restauration de la continuité écologique des cours d'eau (installation de passe à poissons par exemple, pour les migrateurs), et de promotion et labellisation (notamment en appellations locales ou en aquaculture biologique), ou compte s'y mettre lors de futurs programmes (Pönack, 2014). Ainsi, il a été créé un label pour la production de carpes en aquaculture biologique, soutenu par différents partenaires et respectant les critères européens. Le label a en effet été lancé suite à un projet de valorisation de l'aquaculture biologique de carpes en Lusace. Ce projet, porté par le FLAG (fishery local action group) de Haute-Lusace, a bénéficié du soutien de l'Europe via des fonds européens (axe 4 de l'ancien FEP - fonds européen pour la pêche - visant à promouvoir les stratégies locales de développement durable de régions halieutiques), qui ont financé $75 \%$ des $165000 €$ du projet.

Image 2 : logo du label de carpe biologique de Lusace, source : www.biokarpfen.de

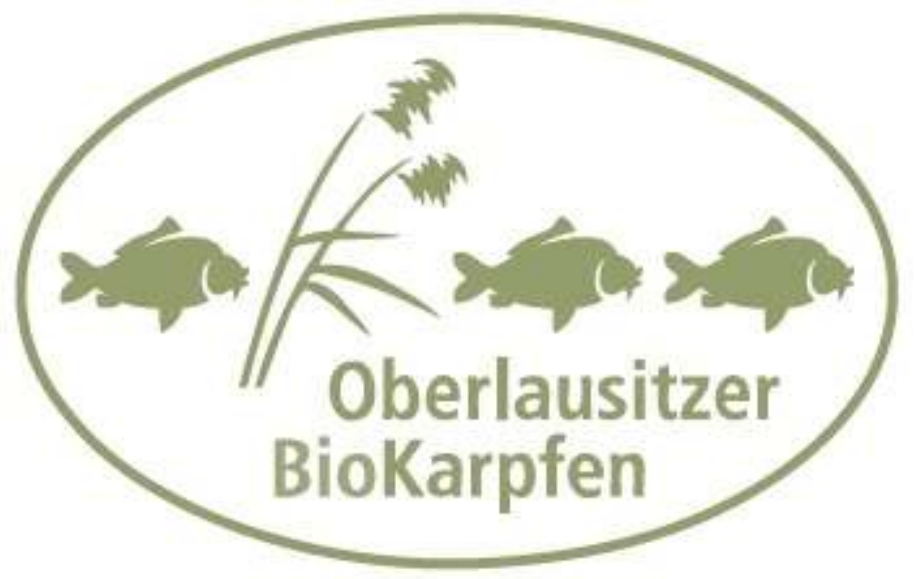

33 La production de carpes biologiques connaît toutefois des débuts difficiles, avec entre 30 et 130 ha d'étangs labellisés suivant les années, et une production en dents de scie : 9 tonnes en 2009, 6 en 2010, 12 en 2011, 10 en 2012, 6 en 2013 (Füllner, Bild \& Matko, op. cit.).

Le classement en réserve de biosphère d'une partie de la Lusace par l'UNESCO dans le cadre de son programme Man \& Biosphere ${ }^{9}$ est également une marque de reconnaissance de la prise en compte de l'environnement sur un territoire, et de sa bonne gestion. Cependant, certains problèmes liés aux protections environnementales apparaissent, comme ailleurs en Europe, et notamment ceux de l'augmentation de populations faunistiques de gros gibiers d'eau par exemple, mais aussi, et c'est problématique pour les ressources piscicoles de Lusace, de l'avifaune (explosion du nombre de couples de cormorans dans les zones humides : aucun présent en 1977, 25000 aujourd'hui). 
La protection des environnements et des ressources aquatiques est donc un pari sur l'avenir que fait la Lusace, contribuant ainsi à l'émergence de nouveaux usages de l'eau, comme le tourisme environnemental, ou la production piscicole de haute qualité et à haute valeur ajoutée, toujours dans un but de développement de l'économie locale.

\section{Conclusion}

La Lusace, région excentrée et peu connue du nord-est de l'Allemagne est un territoire riche de ses milieux aquatiques. De tout temps, les sociétés qui y sont établies ont su tirer profit de l'eau au travers de nombreux usages, et notamment les Sorabes, populations minoritaires d'origine slave. Les usages traditionnels de l'eau sont nombreux, et appartiennent aussi bien au patrimoine matériel qu'immatériel de la région. Leur protection et leur mise en valeur sont assez récentes, et sont utilisées à des fins de développement économique, en cherchant à attirer des touristes avec des événements et sites spécifiques bien identifiables. Cependant, on assiste, en parallèle de la protection, voire du renouveau de ces usages traditionnels de l'eau, à l'apparition d'usages récents pour la région, qui peuvent également s'avérer être des atouts économiques pour le territoire. Ces nouvelles fonctionnalités rurales, pourvoyeuses d'emplois, permettent ainsi de nouveaux débouchés à la population locale, et montrent une volonté forte de la Lusace de mettre en valeur ses paysages et son patrimoine, marqués par la politique économique de la RDA, et les impressionnantes mines de lignite.

\section{BIBLIOGRAPHY}

Conseil de l'Europe, 2004, Charte européenne des langues régionales ou minoritaires, Deuxième rapport périodique présenté au SGCE, Allemagne, Conseil de l'Europe, 305 p.

Duma J., Rzetelska-Feleszko E., 1974, Dolnołuźyckie nazwy rzeczne, Lêtopis ISL, A, 21, 2, pp. 190-205.

Entwicklungsprogramm für den ländlichen Raum in Freistaat Sachsen 2007-2013, 2011, Integriertes Ländliches Entwicklungskonzept (ILEK), " Oberlausitzer Heide- und Teichlandschaft" /

"Hornjołužiska hola a haty", Regionalna mjezyewaluacija 2008 do 2010, UE, EPLR FS, LEADER, 41 p.

Füllner G., 2000, « Fischerei im Biosphärenreservat Oberlausitzer Heide- und Teichlandschaft », in Berichte der Naturforscenden. Gesellschaft Oberlausitz, Band 9, pp. 85-90.

Füllner G., Bild A. \& Matko I., 2013, Aquakultur und Fischerei in Sachsen, Jahresbericht 2013, Landesamt für Umwelt, Landwirtschaft und Geologie, Freistaat Sachsen, 45 p.

Hartstock E., 2000, Entstehung und Entwicklung der Oberlausitzer Teichwirtschaft, Sächsische Landesanstalt für Landwirtschaft, Dresden, $104 \mathrm{p}$.

Koter M., 1995, « Classification génétique des peuples frontaliers d'après l'exemple de la Pologne ", in Goetschy H. et Sanguin A.-L., Langues régionales et relations transfrontalières en Europe, Paris, L'Harmattan, Col. "Géographies et cultures", pp. 235-241. 
Kowar M., 1994, Les Sorabes ou Wendes, Domowina, Bureau d'information sur la culture sorabe, Bautzen, $3 \mathrm{p}$.

Kudela J., 1985, « Pourquoi il n'y a pas eu de République populaire sorabe », in Les Sorabes ou Serbes de Lusace, Paris, INALCO, Civilisations de l'Europe centrale et du Sud-Est, Cahier $n^{\circ} 3$, pp. 85-153.

Kuringowa T., 2005, Les coutumes sorabes au cours de l'année, Serbska kulturna informacija, Załožba za serbski lud, Budyšin, 32 p.

Marti R., 2005, « Dans la gueule du loup : les Sorabes en Allemagne », in Slavica occitania, Toulouse, n²0, pp. 93-112.

Neumann M., 2009, Sorben (Wenden), eine Brandenburger Minderheit und ihre Thematisierung im Unterricht, Universität Potsdam, $107 \mathrm{p}$.

Österreichische Fischereigesellschaft, 2006, Die Namen unserer Fische - eine etymologische Spurensuche : der Schlammpeitzger, 2 p.

Pönack S., 2014, Tourismuskonzept der Stadt Vetschau/Spreewald 2020, Stadt Vetschau/Spreewald / Město Wětošow/Błota, 56 p.

Sanguin A.-L., 1996, « Les Sorabes de l'ex-R.D.A. après la fin du communisme : la recomposition territoriale du plus petit des peuples slaves », in Revue des études slaves, Tome 68, fascicule 1, pp. 55-68.

Scholze B., 2011, Usage et coutumes en Basse-Lusace, Sorbische Kulturinformation Bautzen, 9 p.

Tschernokoshewa E., 2011, Sorbische Identität und Kultur in der Ortslage Proschim (Prožym) mit Karlsfeld, Serbski institut Budyšin, 161 p.

UNESCO, 2011, Qu'est-ce que le patrimoine culturel immatériel ?, UNESCO-PCI, 12 p.

Yèche H., 2014, «Les Sorabes : une minorité invisible ? », in Belgeo [En ligne], 3 | 2013, mis en ligne le 24 mai 2014, consulté le 06 novembre 2014. URL: http://belgeo.revues.org/11570

\section{Filmographie}

Daiber N., 2014, Allemagne : la transition énergétique et les Sorabes, documentaire pour Arte Info, visible en ligne sur info.arte.tv/fr/la-transition-energetique-fait-la-part-belle-au-charbon

\section{NOTES}

1. République Démocratique Allemande

2. Voir le site de l'institut de sorabistique : sorb.philol.uni-leipzig.de

3. Organisation des Nations unies pour l'éducation, la science et la culture

4. DGM e.V. : www.muehlen-dgm-ev.de/index.php

5. WSA : Wasser- und Schifffahrtsamt de Berlin, www.wsa-b.de

6. Voir l'article du Zeit $n^{\circ} 31 / 2012$ : www.zeit.de/2012/31/C-Kahnpostbotin

7. Voir le site de l'association: www.spreewaldfischer-burg.de

8. Voir leur site : www.fischereimuseum.de

9. Voir le site de la réserve : www.biosphaerenreservat-oberlausitz.de 


\section{ABSTRACTS}

This article focuses on traditionnal uses of water in Lusatia, region of the north-east of Germany between the Länder of Brandeburg and Saxony. The hydrographic networkis very denseand numerouspondshave been builtsince medieval times. This areais populated bya slavicminority, theSorbs, who has always usedthewaterfor multiple uses. Water alsoplays an important rolein theSorbiancollective imaginary,and is directlyor indirectlycelebrated atmany festivalsorspecialcustoms.Wateruses arestillperpetuatedin the territory, but their valorizationis fairly recent, while in parallelemergingnew usesof aquatic environmentsconduciveto the economic developmentof the region(tourism, and so on).

Cet article s'intéresse aux usages traditionnels de l'eau en Lusace, région du nord-est allemand située à cheval sur les Länder de Saxe et du Brandebourg. Le réseau hydrographique y est très dense, et de nombreux étangs ont été aménagés depuis l'époque médiévale. Ce territoire est peuplé par une minorité slave, les Sorabes, qui a toujours utilisé l'eau présente pour de multiples usages. L'eau tient aussi une place importante dans l'imaginaire collectif sorabe, et est célébrée directement ou indirectement lors de nombreuses fêtes ou avec des coutumes particulières. Les usages de l'eau sont toujours perpétués sur le territoire, mais leur mise en valeur est assez récente, alors qu'en parallèle émergent de nouveaux usages des milieux aquatiques, propices au développement économique de la région (tourisme, etc).

Dieser Artikel befasst sich auf dietraditionelle Verwendung vonWasser in derLausitz, einer Region im Nordosten Deutschlandsinnerhalb derLänder Sachsenund Brandenburg. DasGewässernetzist sehr dicht undzahlreiche Teichewurdenseit dem Mittelaltergebaut.Dieser Bereich ist voneiner slawischenMinderheit,die Sorben, besiedelt, die das Wasser kontinuierlichfür unterschiedliche Zwecke verwendet hat,Wasser spieltauch eine wichtige Rolleinder sorbischenkollektiven Vorstellungund wirddirekt oder indirektauf vielen Volksfestenoder besondereBräuchegefeiert. Die Wassernutzung wirdnoch heuteim Gebietverewigt, aber ihre Entwicklung istziemlich neu, währenddessen neue Formen der Wassernutzungentstehen und diewirtschaftlicheEntwicklung der Region(Tourismus,usw.) fördern

\section{INDEX}

Schlüsselwörter: Sorben, Lausitz, Teich, Fischerei, Teichwirtschaft

Mots-clés: Sorabes, Lusace, Spree, étang, pêche, pisciculture

Keywords: Sorbs, Lusatia, Spree, pond, fishing, fish farming

\section{AUTHOR}

\section{ANATOLE DANTO}

Etudiant en master 2 d'anthropologieUniversité de Bretagne Occidentale, Institut universitaire européen de la mer, APoliMer - UMR LEMAR 6539, Technopôle Brest-Iroise, 15 rue Dumont d'Urville, 29280 Plouzané - anatole.danto[chez]orange.fr 